\title{
Reformatting the reproductive tract to accommodate the needs of human ARTs
}

\author{
David F. Albertini ${ }^{1}$
}

Published online: 28 February 2017

(C) Springer Science+Business Media New York 2017

Commentary:

Whether viewed as a matter of reformatting or mimicry, ARTs have taken as a singular modus operandi the goal of replicating properties of the female reproductive tract conducive to the journey from ovulation to implantation. From follicular fluid and the gentle stroke of tubal cilia, through peristalsis, and the warm confines of the uterine cavity, gametes, and the products of fertilization interact with an array of environments over a time scale of days. Identifying and recreating the physicochemical properties of this niche and sequence were, historically, the accepted charge first of the food animal science pioneers and later, the cadre of basic scientists who introduced ARTs with experimental laboratory animals like the mouse. Thus, defining the right niche, (at least in chemical form) capable of supporting the early development of embryos has been the dominant objective throughout the evolution of human ARTs [1]. In a sense, the female reproductive tract has been turned inside-out and truncated to suit the logistical clinical management required to realize the follicle to uterus journey.

Along the way, the notion that chemistry alone in the context of embryo metabolism would satisfy the demands of the highest quality embryos suitable for transfer has been viewed as an oversimplification. For example, while details deriving from Leese's "quiet metabolism" hypothesis have been productively adopted by some "media mavericks," those targeting mitochondria (so-called mitomaniacs) are less likely to contextualize the problem in such terms for purposes of embryo selection [2]. At this point, it is safe to say that

David F. Albertini

dalbertini@thechr.com

1 The Center for Human Reproduction, New York, NY, USA recognizing and making appropriate adjustments to culture conditions from detailed animal studies has not been enabled for use in human embryo culture [3].

Such is the case for the embryocentric culture (sic) in human ARTs as we continue down the pathway of a paradigm that simply will not go away - that of understanding and replicating the impact of local microenvironments on the developmental competence of the conceptus. As long as the practice of extended culture retains its current popularity, reformatting efforts, and searching for biomarkers of prognostic value will continue unabated.

What are the targets of study lending credible insights into the problems at hand? Some remain within the realm of the biophysical, drawing upon advances in the field of microfluidics [4]. Others have stagnated as the discourse continues over single or sequential media [5, 6], compositional variants, and their influence on gene expression patterns in media comparisons [7]. In the end, even the question of what stage to transfer-cleavage or blastocyst-lingers while properly executed RCTs loom in a background of mediocrity in terms of clinical decision making [8].

In this vein, we introduce our readership to a dualpurpose set of papers serving to extend the forgoing discussion and bring to light two contributions to our Fellow's Forum Initiative announced earlier this year. The first of these, from Dan Kaser, fittingly sets the stage for what can be anticipated during REI fellowship with respect the sequence of events and challenges encountered during this training experience [9]. Well beyond this intent, his article delivers a spoton perspective for the subject of oxygen tension during embryo culture that is finally receiving the attention it deserves. Taking one step further, Scott Morin then tackles the question of oxygen tension in the context of spatial and temporal changes that the conceptus might be expected to face within confines of the reproductive tract [10]. Interestingly, a recent 
publication identifies oxygen tension as having an important influence on global DNA methylation patterns when embryos cultured at 5 or $20 \%$ were compared [11].

It is our hope that efforts like this will encourage decisive and leading edge research into reproductive medicine from a new generation of specialists aiming to make a difference in the field of human ARTs.

Finally, we welcome Anna Viega and Kui Liu to our editorial board this month, a further sign of our growth and influence on the world wide practice of reproductive medicine.

\section{References}

1. Wale PL, Gardner DK. The effects of chemical and physical factors on mammalian embryo culture and their importance for the practice of assisted human reproduction. Hum Reprod Update. 2016;22(1): $2-22$.

2. Leese HJ. Metabolism of the preimplantation embryo: 40 years on. Reproduction. 2012;143(4):417-27.

3. Baltz JM. Connections between preimplantation embryo physiology and culture. J Assist Reprod Genet. 2013;30(8):1001-7.

4. Swain JE. Shake, rattle and roll: bringing a little rock to the IVF laboratory to improve embryo development. J Assist Reprod Genet. 2014;31(1):21-4.
5. Kleijkers SH, Mantikou E, Slappendel E, Consten D, van EchtenArends J, Wetzels AM, et al. Influence of embryo culture medium (G5 and HTF) on pregnancy and perinatal outcome after IVF: a multicenter RCT. Hum Reprod. 2016;31(10):2219-30.

6. Sfontouris IA, Martins WP, Nastri CO, Viana IG, Navarro PA, Raine-Fenning N, et al. Blastocyst culture using single versus sequential media in clinical IVF: a systematic review and metaanalysis of randomized controlled trials. J Assist Reprod Genet. 2016;33(10):1261-72.

7. Kleijkers SH, Eijssen LM, Coonen E, Derhaag JG, Mantikou E, Jonker MJ, et al. Differences in gene expression profiles between human preimplantation embryos cultured in two different IVF culture media. Hum Reprod. 2015;30(10):2303-11.

8. Glujovsky D, Farquhar C, Quinteiro Retamar AM, Alvarez Sedo CR, Blake D. Cleavage stage versus blastocyst stage embryo transfer in assisted reproductive technology. Cochrane Database Syst Rev. 2016;6:CD002118.

9. Kaser DJ. On developing a thesis for Reproductive Endocrinology and Infertility fellowship: a case study of ultra-low (2\%) oxygen tension for extended culture of human embryos. J Assist Reprod Genet. 2017. doi:10.1007/s10815-017-0887.

10. Morin SJ. Oxygen tension in embryo culture: does a shift to $2 \% \mathrm{O} 2$ in extended culture represent the most physiologic system? J Assist Reprod Genet. 2017. doi:10.1007/s10815-017-0880.

11. Ghosh J, Coutifaris C, Sapienza C, Mainigi M. Global DNA methylation levels are altered by modifiable clinical manipulations in assisted reproductive technologies. Clin Epigenetics. 2017;9:14. 